\title{
Periostin is involved in cell proliferation and interstitial fibrosis in polycystic kidney disease
}

Periostin is overexpressed in autosomal dominant polycystic kidney disease (ADPKD) and in mice with polycystic kidney disease (PKD). Darren Wallace and colleagues at the University of Kansas Medical Center, USA show that decreased expression of periostin reduces the number and size of renal cysts, fibrosis, and improves renal function in mice. Wallace proposed that, "persistent expression of periostin ... in PKD may transmit signals from the extracellular
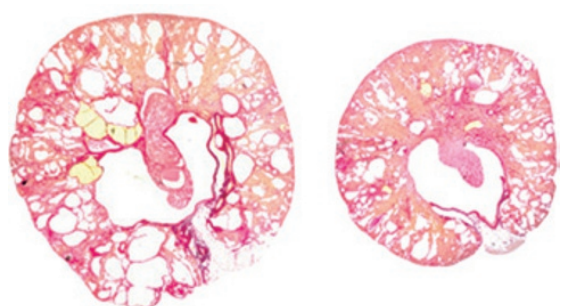

Kidney sections from pcy/pcy:Postn ${ }^{+/+}$(left) and pcy/ pcy:Postn ${ }^{-/-}$(right) mice stained with picrosirius red. Permission obtained from Nature Publishing Group (C) Wallace, D. P. et al. Kidney Int. doi:10.1038/ki.2013.488 matrix ... leading to abnormal cell proliferation and growth of renal cysts," which also suggests that periostin may be a target for therapy in PKD.

Previously, the researchers found that periostin is overexpressed in vitro in epithelial cells lining renal cysts in ADPKD. Periostin is thought to bind integrins on the cell surface, activating integrin-linked kinase (ILK), which leads to accelerated in vitro cell proliferation and the growth of cysts in ADPKD, but not in normal cells.

In the current study, Wallace and his team demonstrate that overexpression of periostin is common to mouse models of renal cystic disease regardless of the underlying genetic mutation. Furthermore, they used the $p c y / p c y$ mouse-a well characterized model of a recessive form of PKD_to determine the effect of periostin expression on disease progression.

Knockout of periostin gene (Postn) expression reduced the number of proliferating cells, the size and number of cysts and decreased renal interstitial fibrosis in $p c y / p c y:$ Post $^{-/-}$ mice. The researchers showed that the loss of periostin expression also improved survival and renal function in these mice compared with those that expressed periostin.

The results indicate that periostin contributes to $\mathrm{PKD}$ progression possibly through an AKT/mTOR signalling pathway activated by ILK. "Periostin contributes to renal cyst enlargement and disease progression in $p c y / p c y$ mice," Wallace continues, "perisotin and possibly other matricellular proteins, and their associated signalling pathways may be viable targets for PKD therapy."

\section{Ellen Bible}

Original article Wallace, D. P. et al. Periostin promotes renal cyst growth and interstitial fibrosis in polycystic kidney disease. Kidney Int. doi:10.1038/ki.2013.488 